\title{
Enfermedad de Kawasaki con manifestaciones asociadas graves y síndrome de activación macrofágica en un paciente pediátrico
} Kawasaki disease with severe manifestations and macrophage activation syndrome in a pediatric patient

\author{
Dra. Natalia Vélez-Tirado ${ }^{a}$ Dr. Gerardo López ${ }^{a}$ Dr. Iván Mijail Chío ${ }^{a}$ y Dr. Alonso Gutiérrez Hernández ${ }^{a}$
}

\section{RESUMEN}

La enfermedad de Kawasaki es una vasculitis febril, aguda y multisistémica, que afecta, principalmente, a niños menores de 5 años. Se describen las características clínicas, la evolución y las consideraciones terapéuticas en un paciente con diagnóstico de enfermedad de Kawasaki completo con manifestaciones multisistémicas graves, dentro de las cuales se resalta el síndrome de activación de macrófagos, que representa una complicación inusual y potencialmente mortal de la enfermedad.

Palabras clave: enfermedad de Kawasaki, meningitis aséptica, apendicitis, sindrome de activación macrofágica.

\section{ABSTRACT}

Kawasaki disease is a febrile, acute and multisystemic vasculitis that mainly affects children under 5 years of age. We describe the clinical characteristics, evolution and therapeutic considerations in a patient with a diagnosis of complete Kawasaki disease with severe multisystem manifestations, among which stands out the macrophage activation syndrome, which represents an unusual and potentially life-threatening complication of the illness. Key words: Kawasaki disease, aseptic meningitis, appendicitis, macrophage activation syndrome.

http:/ / dx.doi.org/10.5546/ aap.2019.e676

Cómo citar: Vélez-Tirado N, López G, Mijail Chío I, Gutiérrez Hernández A. Enfermedad de Kawasaki con manifestaciones asociadas graves y síndrome de activación macrofágica en un paciente pediátrico. Arch Argent Pediatr 2019;117(6):e676-e678. a. Universidad Autónoma de México, México. Instituto Nacional de Pediatría. Servicio de Inmunología y Alergia, Clínica Pediátrica. México D. F.

Correspondencia:

Dra. Natalia Vélez Tirado: Natalia_velezt5@hotmail.com

Financiamiento: Ninguno.

Conflicto de intereses: Ninguno que declarar.

Recibido: 27-1-2019

Aceptado: 26-6-2019

\section{INTRODUCCIÓN}

La enfermedad de Kawasaki (EK) es una vasculitis de la infancia que genera compromiso multisistémico y se asocia a aneurismas coronarios hasta en un $25 \%$ de los casos, porcentaje que disminuye a menos del $5 \%$ tras la administración de gammaglobulina intravenosa (GGIV). Se caracteriza por ser autolimitada y afectar, principalmente, a niños menores de 5 años. ${ }^{1}$

Se ha descrito en todos los países del mundo, con una prevalencia de 243 por cada 100000 niños menores de 5 años en Japón; en Estados Unidos, entre 20 y 25 casos por cada 100000 pacientes, y es mucho mayor en las islas de Hawái respecto de la zona continental. ${ }^{1,2}$ En México, en el Hospital Infantil Federico Gómez, atendieron 204 casos de EK desde 2004 hasta 2014 y, en el Instituto Nacional de Pediatría, 338 casos desde 1995 hasta 2013. ${ }^{3}$

El diagnóstico es clínico. Se considera una EK completa si el paciente presenta más de 5 días de fiebre, además de 4 de los siguientes 5 criterios: 1. eritema de los labios, labios fisurados, lengua en frambuesa y / o eritema de la mucosa oral; 2. conjuntivitis bilateral no purulenta; 3. exantema maculopapular, eritrodermia difusa o exantema similar al eritema multiforme; 4 . eritema y edema de manos y pies en la fase aguda o descamación en la fase subaguda; 5 . adenopatía cervical, unilateral, mayor de $1,5 \mathrm{~cm} .^{1}$

\section{CASO CLÍNICO}

Paciente masculino de 5 años de edad previamente sano y sin antecedentes de importancia, que presentó fiebre de $39,7^{\circ} \mathrm{C}$, dolor abdominal periumbilical punzante con posterior presencia de eritema en los labios y placas eritematosas con descamación fina en la comisura labial. Acudió a Medicina General y se le inició el esquema antibiótico con diagnóstico de faringoamigdalitis bacteriana. El dolor abdominal tuvo una lenta migración hacia la fosa ilíaca derecha, por lo que, al quinto día, decidió consultar al Servicio de Urgencias. 
Al ingresar, presentó fiebre persistente de alto grado, conjuntivitis bilateral no exudativa, hiperemia en los labios, queilitis, lengua en frambuesa, cuello con adenomegalia lateral derecha de $0,5 \times 1 \mathrm{~cm}$, exantema en la región torácica y dorsal, eritema en las palmas y las plantas. Presentó dolor localizado en la fosa iliaca derecha, con signos de irritación peritoneal y hepatoesplenomegalia. Se realizó un ultrasonido abdominal, en el que se observó líquido libre en la fosa ilíaca derecha, sin presencia de hidrocolecisto, con hepatoesplenomegalia leve, con signos ecográficos compatibles con un proceso inflamatorio apendicular, por lo que se decidió realizar apendicectomía laparoscópica.

Se hizo el diagnóstico de EK. Se realizó un ecocardiograma, que no mostró anormalidades estructurales; el pro péptido natriurético tipo B se reportó en $790 \mathrm{mg} / \mathrm{dl}$ (menor de $300 \mathrm{mg} / \mathrm{dl}$ en menores de 50 años). Al ingresar, el hemograma reportó hemoglobina: 14,4 g/ dl (10,7-14,7 mg/ dl); hematocrito: el 40,5\% (el 35-42\%); leucocitos: 3400 / $\mu 1$ (5500-15 500/ $\mu$ l; neutrófilos: 3100 / $\mu 1$ (1500-8500); linfocitos: 300/ $\mu 1$ (2000-8000/ $\mu 1)$ plaquetas: $41000 / \mu 1(150000-450000 / \mu 1)$; proteína $C$ reactiva (PCR): $8 \mathrm{mg} / \mathrm{dl}$ (normal: menor de $1 \mathrm{mg} / \mathrm{dl}$ ); velocidad de sedimentación globular: $2 \mathrm{~mm} / 1 \mathrm{~h}$.

Debido al dolor abdominal y a la hepatomegalia, se tomó la función hepática, que reportó bilirrubina total: $1,51 \mathrm{mg} / \mathrm{dl}(0,2-$ $1 \mathrm{mg} / \mathrm{dl})$; bilirrubina directa: $0,71 \mathrm{mg} / \mathrm{dl}(0,0-$ $0,3 \mathrm{mg} / \mathrm{dl})$; bilirrubina indirecta: $0,8 \mathrm{mg} / \mathrm{dl}$ (0,0-0,7 mg/dl); albúmina: 3,3 g/ dl (3,5-4 g/ dl); fosfatasa alcalina: $239 \mathrm{U} / 1$ (50-136 U/1); lactato deshidrogenasa: $979 \mathrm{U} / 1$ (190-390 U/1); aspartato aminotransferasa: 274 U/1 (1537 U/1); alanino aminotransferasa: 105 U/1 (30$65 \mathrm{U} / \mathrm{l})$. Se administraron $2 \mathrm{~g} / \mathrm{kg}$ de GGIV al $10 \%$; no se inició ácido acetilsalicílico por la presencia de trombocitopenia. 36 horas tras la administración de GGIV, el paciente persistió con fiebre, somnolencia progresiva alternante con hipoactividad e irritación meníngea.

Se realizó una tomografía cerebral simple, que descartó lesiones que ocuparan espacio o un edema cerebral. Se tomó una punción lumbar con proteínas: $179 \mathrm{mg} / \mathrm{dl}(20-45 \mathrm{mg} / \mathrm{dl})$; glucosa: 42,6 mg/dl (42-65 mg/dl); 38 células $\mathrm{mm}^{3}\left(0-6 \mathrm{~mm}^{3}\right)$; polimorfonucleares: el $10 \%$ (el $0 \%$ ); mononucleares: el $90 \%$; la reacción en cadena de la polimerasa para 13 patógenos, así como los cultivos, fueron negativos; el electroencefalograma se reportó como normal.
Con lo anterior, se consideró que presentaba resistencia al tratamiento con GGIV y se administró una nueva dosis de GGIV a razón de $2 \mathrm{~g} / \mathrm{kg}$. A las 24 horas después de la segunda dosis de GGIV, el paciente continuó febril, con linfocitos de $300 / \mu 1$ y plaquetas de $23000 / \mu 1$.

Por la sospecha de SAM, se solicitó ferritina, que reportó $1700 \mathrm{ng} / \mathrm{ml}$ (7-142 ng/ $\mathrm{ml}$ ), triglicéridos de $190 \mathrm{mg} / \mathrm{dl}$ (menor de $150 \mathrm{mg} / \mathrm{dl}$ ) y fibrinógeno de $110 \mathrm{mg} / \mathrm{dl}$ (200$400 \mathrm{mg} / \mathrm{dl}$ ). Se inició el tratamiento con pulsos de metilprednisolona a razón de $30 \mathrm{mg} / \mathrm{kg} /$ día por dos días seguidos, con la desaparición de la fiebre, la normalización del recuento de linfocitos y plaquetas, el descenso progresivo de parámetros inflamatorios, la normalización de la hepatoesplenomegalia y la regularización de la función hepática. Tras la mejoría del recuento plaquetario, se inició un manejo antiagregante con ácido acetilsalicílico a razón de $5 \mathrm{mg} / \mathrm{kg} /$ día, así como metilprednisolona a razón de $1 \mathrm{mg}$ / kg/ día. La biopsia de apéndice cecal reportó periapendicitis mononuclear leve.

En su seguimiento, el paciente ha tenido buena evolución. Desde su ingreso y durante el año que lleva de seguimiento, no ha desarrollado complicaciones. De igual forma, permitió el descenso de esteroide hasta su suspensión completa.

\section{DISCUSIÓN}

En la literatura, hay pocos reportes de pacientes con EK que presenten múltiples complicaciones graves, como vasculitis abdominal, meningitis aséptica y SAM. La EK puede generar compromiso abdominal; la manifestación gastrointestinal más frecuente es la alteración de la función hepática.

Singh et al. reportaron a 280 pacientes, de los cuales el $30 \%$ tenía elevación moderada de las transaminasas $\mathrm{y}$, ocasionalmente, ictericia obstructiva secundaria a hidropesía de la vesícula biliar. ${ }^{4}$ La vasculitis periapendicular y la apendicitis son manifestaciones poco comunes que requieren la evaluación y el manejo oportuno por Cirugía Pediátrica para disminuir las complicaciones. ${ }^{5}$

Zulian et al. revisaron a 219 pacientes con EK en 2 centros reumatológicos en Italia y encontraron que el 4,5\% de los niños tenían abdomen quirúrgico agudo. También reportaron obstrucción intestinal, pseudo obstrucción intestinal, pancreatitis, colitis isquémica, duodenitis hemorrágica y necrosis hepática. ${ }^{6}$ 
Dentro de las manifestaciones del sistema nervioso central, la más común es la meningitis aséptica (el $25 \%$ ), crisis convulsivas, ataxia, parálisis facial e infarto cerebral (menos del $1 \%)^{7,8}$

Kemmotsu et al. buscaron diferenciar los casos de meningitis aséptica por EK de los inducidos por GGIV. Encontraron que la asociada a GGIV aparecía en las primeras $48 \mathrm{~h}$ tras la administración del medicamento y tenía infiltrado con predominio polimorfonuclear en el análisis del líquido cefalorraquídeo (LCR). Además, la meningitis secundaria a EK se presentaba más temprano en el curso de la enfermedad y tenía un infiltrado de LCR con predominio mononuclear ${ }^{9}$ Dado el tiempo de presentación y el análisis del LCR, se consideró que el paciente padecía meningitis aséptica por EK.

El SAM es una complicación infrecuente, pero grave y potencialmente mortal, que requiere diagnóstico temprano y tratamiento apropiado. García-Pavón et al. revisaron los casos de 69 pacientes con EK y SAM en diferentes países y encontraron una incidencia aproximada del 1,1\%. En este grupo, 34 de 69 pacientes eran mayores de 5 años, lo cual podría sugerir una edad mayor como un factor predisponente para SAM. ${ }^{10}$

Se debe sospechar SAM en todo paciente con fiebre persistente, citopenias, fibrinógeno bajo, ferritina elevada, esplenomegalia, triglicéridos elevados, $y$, aunque no es un criterio diagnóstico, la presencia de velocidad de sedimentación globular disminuida es sugestiva de activación macrofágica. ${ }^{10}$ Los glucocorticoides en dosis alta de $30 \mathrm{mg} / \mathrm{kg} /$ día son una aproximación terapéutica segura que suele ser suficiente en este grupo de pacientes para controlar la actividad de la enfermedad.

En los pacientes con EK, se requiere un alto índice de sospecha para detectar complicaciones graves, inusuales y potencialmente mortales, como lo es el SAM.

\section{Agradecimientos}

A Juan Esteban Cote por su apoyo incondicional.

\section{REFERENCIAS}

1. McCrindle BW, Rowley AH, Newburger JW, Burns JC, et al. Diagnosis, treatment, and long-term management of Kawasaki disease: A scientific statement for health professionals from the American Heart Association. Circulation. 2017;135(17):e927-99.

2. Singh S, Jindal AK, Pilania RK. Diagnosis of Kawasaki disease. Int J Rheum Dis. 2018;21(1):36-44.

3. Garrido-García LM, Soto-Blanquel JL, Espinosa-Rosales FJ. Enfermedad de Kawasaki: cuadro clínico, exámenes de laboratorio y lesiones coronarias. Acta Pediatr Méx. 2015;36(4):314-21.

4. Singh R, Ward C, Walton M, Persad R. Atypical Kawasaki disease and gastrointestinal manifestations. Paediatr Child Health. 2007;12(3):235-7.

5. Garnett GM, Kimball S, Melish ME, Thompson KS, et al. Appendicitis as the presenting manifestation of Kawasaki disease. Pediatr Surg Int. 2014;30(5):549-52.

6. Trapani S, Montemaggi A, Simonini G, Calabri GB, et al. Surgical abdomen with intestinal pseudo-obstruction as presenting feature of atypical Kawasaki disease. J Paediatr Child Health. 2016;52(11):1032-4.

7. Martínez-Guzmán E, Gámez-González LB, RivasLarrauri F, Sorcia-Ramírez G, Yamazaki-Nakashimada M. Manifestaciones neurológicas en la enfermedad de Kawasaki atípica. Rev Alerg Mex. 2017;64(3):376-80.

8. Rodríguez-González M, Castellano-Martínez A, PérezReviriego AA. Atypical Presentation of Incomplete Kawasaki Disease: A Peripheral Facial Nerve Palsy.J Emerg Med. 2018;55(1):118-20.

9. Kemmotsu Y, Nakayama T, Matsuura H, Saji T. Clinical characteristics of aseptic meningitis induced by intravenous immunoglobulin in patients with Kawasaki disease. Pediatr Rheumatol Online J. 2011;9:28.

10. García-Pavón S, Yamazaki-Nakashimada MA, Báez M, Borjas-Aguilar KL,MurataC. KawasakiDiseaseComplicated With Macrophage ActivationSyndrome: asystematicreview. J Pediatr Hematol Oncol. 2017;39(6):445-51. 